\title{
An Improved Method of Retrieving Sea Surface Wind Speed Based on a Four-Layer Medium Model at High Sea States
}

\author{
Jiasheng Tian*, Qiaoyun Liu, Wan Pan, Jian Shi \\ The Department of Electronics and Information Engineering, \\ Huazhong University of Science and Technology, Wuhan, China \\ Email: ${ }^{*} \underline{\text { ianjs@mail.hust.edu.cn }}$
}

Received June 23, 2013; revised July 28, 2013; accepted August 22, 2013

Copyright (C) 2014 Jiasheng Tian et al. This is an open access article distributed under the Creative Commons Attribution License, which permits unrestricted use, distribution, and reproduction in any medium, provided the original work is properly cited. In accordance of the Creative Commons Attribution License all Copyrights (C) 2014 are reserved for SCIRP and the owner of the intellectual property Jiasheng Tian et al. All Copyright (C) 2014 are guarded by law and by SCIRP as a guardian.

\section{ABSTRACT}

Considering about the effect of whitecaps and foams on pulse-limited Radar Altimeters, an improved algorithm of retrieving sea surface wind speed is proposed in this paper. Firstly, a four-layer dielectric model is established in order to simulate an air-sea interface. Secondly, the microwave reflectivity of a sea surface covered by spray droplets and foams at $13.5 \mathrm{GHz}$ is computed based on the established model. These computed results show that the effect of spray droplets and foams in high sea state conditions shall not be negligible on retrieving sea surface wind speed. Finally, compared with the analytical algorithms proposed by Zhao and some calculated results based on a three-layer dielectric model, an improved algorithm of retrieving sea surface wind speed is presented. At a high wind speed, the improved algorithm is in a better accord with some empirical algorithms such as Brown, Young ones and et al., and also in a good agreement with ZT and other algorithms at low wind speed. This new improved algorithm will be suitable not only for low wind speed retrieval, but also for high wind speed retrieval. Better accuracy and effectiveness of wind speed retrieval can also be obtained.

\section{KEYWORDS}

Satellite Altimeter; Wind Speed Retrieving Algorithm; High Sea States; Stratified Media; Whitecap Coverage Rate

\section{Introduction}

Satellite radar altimetry research project could date back to the conference of solid earth and ocean physics held in Williamstown in 1969. After the recent decades, the altimeter has evolved through Skylab [1], GEOS-3 [2], Seasat and Geosat, Topex/Poseidon and ERS-1 missions. However, the retrieval of the sea surface wind speed from the radar altimeter data poses a great challenge due to the accuracy requirement set forth by the oceanographic research community and some applications, such as wind speed measurement with an accuracy of $2 \mathrm{~m} / \mathrm{s}$, especially in high sea state conditions.

Historically, almost all the altimeter wind speed retrieving algorithms (or mode functions) were based on

"Corresponding author. the relationship between the backscattering coefficient $\sigma^{0}$ and the neutral stable wind speed $U_{10}$ at 10 meters high above sea level. A large number of wind speed retrieving algorithms has been published from 1950s [3-7]. Almost all low speed mode functions tend to overlap when the wind speed is about $U_{10}=10 \sim 7 \mathrm{~m} / \mathrm{s}$. This reason is that sea states are mainly related to the maximum probability of 10 7 m/s wind speed. The second phenomenon is that some high wind speed model functions converge near $9.5 \mathrm{~m} / \mathrm{s}$, but spread quickly when the wind speed is more than $9.5 \mathrm{~m} / \mathrm{s}$. The discrete situation is mainly because most wind speed retrieving algorithms only play an emphasis on the scattering coefficient $\sigma^{0}$ and neglect wave states (significant wave height, the wave age, etc.) [8-10]. Moreover, for those presented functions, some other factors such as spray droplets, 
foams and whitecap coverage rate [11] caused by a cyclone or hurricane were also not considered about.

In 1983, Q. A. Zheng [11] computed the reflectivity of a sea surface covered by whitecaps and foams at 13.9 $\mathrm{GHz}$ based on electromagnetic field theory of stratified media, and studied the effect of oceanic whitecaps and foams on pulse-limited radar altimeters. In 1986, Gairola [12] also investigated the reflectivity of a sea surface covered by whitecaps and foams at $13.9 \mathrm{GHz}$ based on a three-layer medium model, and applied the computed result to the sea surface wind speed retrieval. In 2008, Yang [13] also computed a three-layer model for correcting backscattering coefficient. When these research results were applied in retrieving sea surface wind speed, the accuracy would be improved to some extent. However, in high sea state conditions, some differences from Young algorithm or Brown's would still exist.

In 2003, Zhao and Toba proposed an analysis algorithm (referred to as ZT) [6,7] considering about the effect of wave states (or sea states). The algorithm was derived from electromagnetic wave scattering theory and wave spectral theory, and was independent of the spatial and temporal registration data, the size of the measured data and the spatial and temporal data registration standards and so on. The algorithm took the role of wave age into account in retrieving wind speed, and thus its symmetry was good and its root mean square error could also be accepted. However, for this algorithm, the effect of sea foams and spray droplets was not taken into account. The reflectivity $|R(0)|^{2}$ in the expression of the $\mathrm{ZT}$ function should not be considered to be generated only by the sea water, but a hybrid interface consisting of multilayer media including sea water, foams, spray droplets, the air.

The purpose of this study is to discuss the effect of whitecaps, spray droplets and foams on measurements of wind speed on pulse-limited radar altimeters by calculating quantitatively the microwave reflectivity of a foamcovered sea surface based on a four-layer-medium model. The calculated results will be applied into retrieving sea surface wind speed at high sea states.

\section{Electromagnetic Scattering from the Sea Surface}

\subsection{Physical Model}

The approach for measuring sea surface wind speed by radar altimeter is based on the theory that the sea surface backscattering coefficient $\sigma^{0}$ is a function of the wind speed. Namely, the usual wind speed retrieval algorithm is based on the direct relationship between wind speed and backscattering coefficient $\sigma^{0}$. Based on the specular point theory almost all algorithms (including empirical, semi-empirical or analytic algorithms) can be ex- pressed as $[14,15]$

$$
\sigma^{0}=\pi|R(\theta)|^{2} \sec ^{4} \theta p\left(\zeta_{x}, \zeta_{y}\right)
$$

where $p\left(\zeta_{x}, \zeta_{y}\right)$ is the probability density function of sea surface mean square slopes $\bar{S}^{2}$ relating to wind speed. $|R(\theta)|^{2}$ is the reflectivity of the air-to-surface interface at the incident angle $\theta$. The wind speed is related not only to mean square slopes $\bar{S}^{2}$ but also to the air-sea interface reflectivity factor $|R(\theta)|^{2}$. Under the classical assumption that the sea surface mean squre slopes are nearly Gaussian and isotropic in their distribution, the scattering coefficient is given by

$$
\sigma^{0}(\theta)=\frac{|R(\theta)|^{2}}{\bar{S}^{2}} \sec (\theta) \exp \left(-\tan ^{2}(\theta) / \bar{S}^{2}\right)
$$

In fact the sea surface process is more complex. In high sea states, sea wave is broken with the high wind speed, an air-sea interface will become a multilayer medium that is made of air, spray droplets(or droplets), foams, and sea water. It is also known that the thickness of the foam and the droplets layer and the coverage rate will vary with the wind speed [16,17]. In 1982, Zheng studied sea foams influence on electromagnetic wave reflection $|R(0)|^{2}$ at a normal incident wave, and pointed out that the effect of foams and whitecaps on wave reflection should not be insignificant. However zheng or Gairola only computed the microwave reflectivity based on a three-layer medium made of air, foams and sea water, and the effect of the spray droplets was neglected. In order to improve the accuracy of estimated wind speed, it is necessary to establish the electromagnetic wave incidence-reflection physical model in high sea state conditions. Based on the above analysis and experience, in high sea state conditions the air-sea surface should be made up of a four-layer media (Figure 1). The top layer is atmosphere (or air), followed by the sea spray droplet layer. The third layer is foams and the bottom is the seawater layer (Figure 1). The electromagnetic wave from an altimeter penetrates into the air, and enters into the spray droplet layer and foams, finally plunges upon the sea water. The echo from the four-layer medium will be received by the altimeter. $\theta$ stands for the incident angle, $d_{1}$ and $d_{2}$ is the thickness of the droplets and foam layer (Figure 1), respectively.

\subsection{Electromagnetic Scattering Theory}

\subsubsection{TE Wave}

Let's suppose that the electric field intensity of an incident wave with the vertical polarization is given as

$$
\mathbf{E}=\mathbf{a}_{y} \mathbf{E}_{y}=\mathbf{a}_{y} E_{0} \mathrm{e}^{-j \mathbf{k} \cdot \mathbf{r}}=\mathbf{a}_{y} E_{0} \mathrm{e}^{-j k_{0} x \sin \theta-j k_{0} z \cos \theta}
$$

and plunges into the four-layer medium at incident angle $\theta$. $\boldsymbol{a}_{y}$ is the unit vector along $y$ direction, $k_{0}$ is the wave 


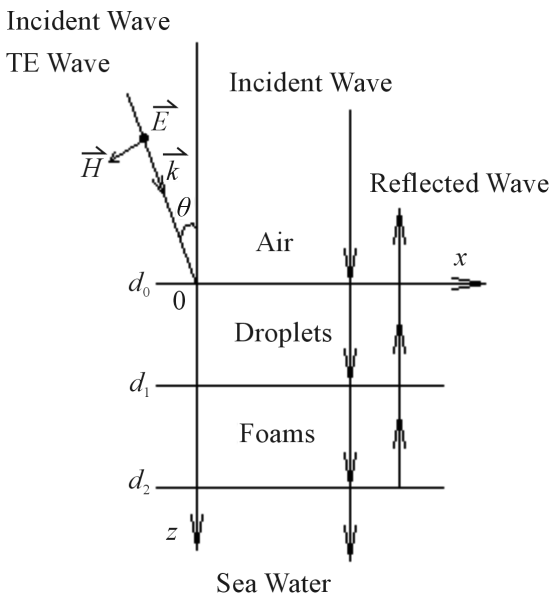

(a)

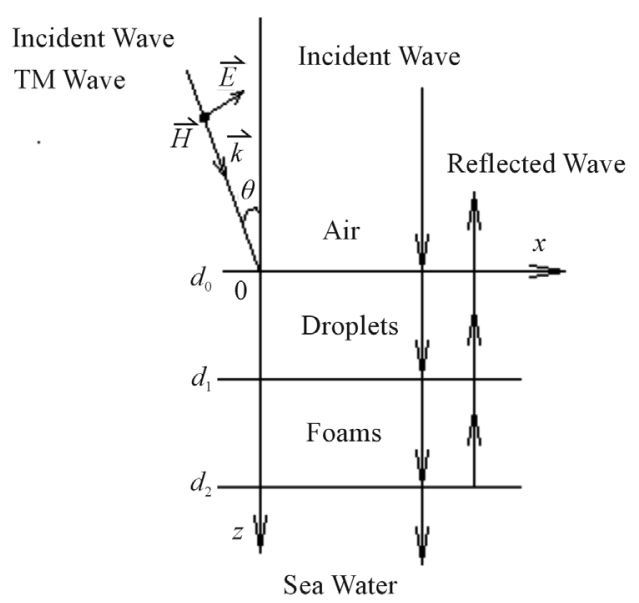

(b)

Figure 1. A multilayer dielectric incident-reflected model. (a) TE wave; (b) TM wave.

number in free space. According to electromagnetic theory, electromagnetic waves in the four-layer media can be expressed as the form of the sum of incident wave and reflected wave, that is

$$
\mathbf{E}=\mathbf{a}_{y} E_{y m}=\mathbf{a}_{y}\left(E_{m}^{+} \mathrm{e}^{-j k_{z m} z}+E_{m}^{-} \mathrm{e}^{j k_{z m} z}\right) \mathrm{e}^{-j k_{m} x \sin \theta_{m}}
$$

where $m=0,1,2,3$ represent the air layer, the droplets layer, the foam layer and the sea water layer respectively. When $m=3$, the second term of Equation (3) is zero in the sea water layer. There $k_{m}=\omega \sqrt{\mu_{m} \varepsilon_{m}}, \mu_{m}$ and $\varepsilon_{m}$ are the permeability and permittivity, respectively. According to the Maxwell equations, we can get the magneticfield components given as

$$
\begin{gathered}
H_{x m}=\frac{-k_{z m}}{\omega \mu_{m}}\left(E_{m}^{+} \mathrm{e}^{-j k_{z m} z}-E_{m}^{-} \mathrm{e}^{j k_{z m} z}\right) \mathrm{e}^{-j k_{m} x \sin \theta_{m}} \\
H_{z m}=\frac{k_{m} \sin \theta_{m}}{\omega \mu_{m}}\left(E_{m}^{+} \mathrm{e}^{-j k_{z m} z}+E_{m}^{-} \mathrm{e}^{j k_{z m} z}\right) \mathrm{e}^{-j k_{m} x \sin \theta_{m}}
\end{gathered}
$$

where $\theta_{m}$ is an angle of incidence or refraction. Ob- viously

$$
\begin{gathered}
k_{0} \sin \theta_{0}=k_{m} \sin \theta_{m}, m=1,2,3 \\
k_{z m}^{2}=k_{m}^{2}-\left(k_{0} \sin \theta_{0}\right)^{2}, m=1,2,3
\end{gathered}
$$

according to electromagnetic theory and the boundary condition which the electromagnetic field tangential components are continuous at the interface, we can get

$$
\left(\begin{array}{c}
E_{y m}^{+} \mathrm{e}^{-j k_{z m} d_{m}} \\
E_{y m}^{-} \mathrm{e}^{j k_{z m} d_{m}}
\end{array}\right)=N_{m(m+1)}\left(\begin{array}{c}
E_{y(m+1)}^{+} \mathrm{e}^{-j k_{z(m+1)} d_{(m+1)}} \\
E_{y(m+1)}^{-} \mathrm{e}^{j k_{z(m+1)} d_{(m+1)}}
\end{array}\right)
$$

where

$$
\begin{array}{ll}
N_{m(m+1)}=\frac{1}{2}\left(1+\frac{\mu_{m} k_{z(m+1)}}{\mu_{(m+1)} k_{z m}}\right) & \\
\cdot\left(\begin{array}{cc}
\mathrm{e}^{j k_{z(m+1)}\left(d_{(m+1)}-d_{m}\right)} & R_{T E, m(m+1)} \mathrm{e}^{-j k_{z(m+1)}\left(d_{(m+1)}-d_{m}\right)} \\
R_{T E, m(m+1)} \mathrm{e}^{j k_{z(m+1)}\left(d_{(m+1)}-d_{m}\right)} & \mathrm{e}^{-j k_{z(m+1)}\left(d_{(m+1)}-d_{m}\right)}
\end{array}\right)
\end{array}
$$

and

$$
R_{T E, m(m+1)}=\frac{1-\frac{\mu_{m} k_{z(m+1)}}{\mu_{(m+1)} k_{z m}}}{1+\frac{\mu_{m} k_{z(m+1)}}{\mu_{(m+1)} k_{z m}}}, m=0,1,2
$$

Thus for the four-layer model (Figure 1), we have

$$
\left(\begin{array}{c}
E_{y 0}^{+} \mathrm{e}^{-j k_{z 0} d_{0}} \\
E_{y 0}^{-} \mathrm{e}^{j k_{z 0} d_{0}}
\end{array}\right)=N_{01} N_{12} N_{23}\left(\begin{array}{c}
E_{y 3}^{+} \mathrm{e}^{-j k_{z 3} d_{3}} \\
E_{y 3}^{-} \mathrm{e}^{j k_{z 3} d_{3}}
\end{array}\right)
$$

Because the thickness of the sea water layer is infinite and there may be considered no reflection wave, we can let $d_{3}=0$ and $E_{y 3}^{-}=0$, and obtain the ratio $R_{T E}$ of the reflected wave to the incident wave at $z=0$ (Figure $1(\mathrm{a})$ ).

$$
R_{T E}=\frac{E_{0}^{-}}{E_{0}^{+}}=\frac{R_{\text {up } 1}}{R_{\text {low } 1}}
$$

where

$$
\begin{aligned}
R_{u p 1}= & R_{T E, 01}+R_{T E, 01} R_{T E, 12} R_{T E, 23} \mathrm{e}^{-j 2 k_{z 2} d_{2}} \\
& +R_{T E, 12} \mathrm{e}^{-j 2 k_{z 1} d_{1}}+R_{T E, 23} \mathrm{e}^{-j 2 k_{z 1} d_{1}-j 2 k_{z 2} d_{2}}
\end{aligned}
$$

and

$$
\begin{aligned}
R_{\text {low } 1}=1 & +R_{T E, 12} R_{T E, 23} \mathrm{e}^{-j 2 k_{z 2} d_{2}}+R_{T E, 01} R_{T E, 12} \mathrm{e}^{-j 2 k_{z 1} d_{1}} \\
& +R_{T E, 01} R_{T E, 23} \mathrm{e}^{-j 2 k_{z 1} d_{1}-j 2 k_{z 2} d_{2}}
\end{aligned}
$$

$d_{1}, d_{2}$ is the thickness of the droplets layer, the foam layer at high wind speed, respectively. $d_{1}$ and $d_{2}$ has the relationship with the wind speed $U_{10}$ at 10 meters height over the sea surface. They can be given as (Andreas E. L., 1995; Wu Jin, 1979) [16,17]. 


$$
\begin{gathered}
d_{1}=0.0075 U_{10}^{2} \\
d_{2}= \begin{cases}0.004 & U_{10} \leq 7 \mathrm{~m} / \mathrm{s} \\
0.004+\left(U_{10}-7\right) \times 0.0012 & U_{10}>7 \mathrm{~m} / \mathrm{s}\end{cases}
\end{gathered}
$$

From Equation (12), reflection characteristics of the air-sea interface can be calculated.

\subsubsection{TM Wave}

Let's suppose that the magnetic field intensity of an incident wave with the parallel polarization is given as

$$
\mathbf{H}=\mathbf{a}_{y} H_{y}=\mathbf{a}_{y} H_{y 0} \mathrm{e}^{-j k_{0} \sin \theta x-j k_{0} \cos \theta z}
$$

Similarly, the magnetic field intensity in the four-layer media can be expressed as the form of the sum of incident wave and reflected wave

$$
\begin{aligned}
\mathbf{H}_{m} & =\mathbf{a}_{y} H_{y m} \\
& =\mathbf{a}_{y}\left(H_{y m}^{+} \mathrm{e}^{-j k_{z m} z}+H_{y m}^{-} \mathrm{e}^{j k_{z m} z}\right) \mathrm{e}^{-j k_{0} \sin \theta x}
\end{aligned}
$$

In the sea water layer $(m=3)$, the second term of Equation (16) is zero. From Maxwell's equation, we can get the electric field intensity. In term of electromagnetic field boundary conditions, we can have

$$
\left(\begin{array}{c}
H_{y m}^{+} \mathrm{e}^{-j k_{z m} d_{m}} \\
H_{y m}^{-} \mathrm{e}^{j k_{z m} d_{m}}
\end{array}\right)=M_{m(m+1)}\left(\begin{array}{c}
H_{y(m+1)}^{+} \mathrm{e}^{-j k_{z(m+1)} d_{(m+1)}} \\
H_{y(m+1)}^{-} \mathrm{e}^{j k_{z(m+1)} d_{(m+1)}}
\end{array}\right)
$$

where

$$
\begin{aligned}
& M_{m(m+1)}=\frac{1}{2}\left(1+\frac{\varepsilon_{m} k_{z(m+1)}}{\varepsilon_{(m+1)} k_{z m}}\right) \\
& \cdot\left(\begin{array}{cc}
\mathrm{e}^{j k_{z(m+1)}\left(d_{(m+1)}-d_{m}\right)} & R_{m(m+1)} \mathrm{e}^{-j k_{z(m+1)}\left(d_{(m+1)}-d_{m}\right)} \\
R_{m(m+1)} \mathrm{e}^{j k_{z(m+1)}\left(d_{(m+1)}-d_{m}\right)} & \mathrm{e}^{-j k_{z(m+1)}\left(d_{(m+1)}-d_{m}\right)}
\end{array}\right)
\end{aligned}
$$

and

$$
R_{T M, m(m+1)}=\frac{1-\frac{\varepsilon_{m} k_{z(m+1)}}{\varepsilon_{(m+1)} k_{z m}}}{1+\frac{\varepsilon_{m} k_{z(m+1)}}{\varepsilon_{(m+1)} k_{z m}}}
$$

Thus for the four-layer-model (Figure 1), we have

$$
\left(\begin{array}{c}
H_{y 0}^{+} \mathrm{e}^{-j k_{z 0} d_{0}} \\
H_{y 0}^{-} \mathrm{e}^{j k_{z 0} d_{0}}
\end{array}\right)=M_{01} M_{12} M_{23}\left(\begin{array}{c}
H_{y 3}^{+} \mathrm{e}^{-j k_{z 3} d_{3}} \\
H_{y 3}^{-} \mathrm{e}^{j k_{z 3} d_{3}}
\end{array}\right)
$$

Letting $d_{3}=0$ and $H_{y 3}^{-}=0$, we can obtain the ratio $R_{T M}$ of the reflected wave to the incident wave at $z=0$ (Figure 1(b)).

$$
R_{T M}=\frac{H_{0}^{-}}{H_{0}^{+}}=\frac{R_{\text {up } 2}}{R_{\text {low } 2}}
$$

where

$$
\begin{aligned}
R_{u p 2}= & R_{T M, 01}+R_{T M, 01} R_{T M, 12} R_{T M, 23} \mathrm{e}^{-j 2 k_{z 2} d_{2}} \\
& +R_{T M, 12} \mathrm{e}^{-j 2 k_{z 1} d_{1}}+R_{T M, 23} \mathrm{e}^{-j 2 k_{z 1} d_{1}-j 2 k_{z 2} d_{2}}
\end{aligned}
$$

and

$$
\begin{aligned}
R_{\text {low } 2}= & 1+R_{T M, 12} R_{T M, 23} \mathrm{e}^{-j 2 k_{z 2} d_{2}}+R_{T M, 01} R_{T M, 12} \mathrm{e}^{-j 2 k_{z 1} d_{1}} \\
& +R_{T M, 01} R_{T M, 23} \mathrm{e}^{-j 2 k_{z 1} d_{1}-j 2 k_{z 2} d_{2}}
\end{aligned}
$$

From Equation (21), we can discuss the reflection characteristics of the four-layer-medium for TM wave.

\subsection{Some Discussions about the Reflected Wave from the Sea Surface}

In terms of Equations (12) and (21), four curves $\left(\theta=0^{\circ}\right.$, $\left.5^{\circ}, 10^{\circ}, 15^{\circ}\right)$ of the reflectivity $\left|R_{T E}\right|^{2},\left|R_{T M}\right|^{2}$ versus the wind speed were plotted (Figure 2). The computed results show that the power reflectivity oscillates when the wind speed is less than $5 \mathrm{~m} / \mathrm{s}$ or the thickness of spray droplets and foams is less than $0.2 \mathrm{~m}$ with the thickness of foams being $0.004 \mathrm{~m}$. Those minmax points (Figure 2) are caused by the resonant absorption of the spray droplets layer and the foams layer. When the wind speed is more than $5 \mathrm{~m} / \mathrm{s}$, The reflectivity is 0.236 or so. Although the curves (Figure 2) are obtained under some assumed conditions (e.g., the incident angle is assumed to be $0^{\circ}, 5^{\circ}$, the temperature is $20^{\circ} \mathrm{C}$.), the curves may represent typical ocean conditions. For a radar altimeter, the incident angle is often less than $5^{\circ}$, so we can draw a conclusion:

$$
\begin{aligned}
\left|R_{T M}(\theta)\right|^{2} & =0.2347 \approx\left|R_{T E}(\theta)\right|^{2} \\
& =0.2372 \approx|R(0)|^{2}=0.2360
\end{aligned}
$$

when $\theta$ is less than $5^{\circ}$, the difference will cause an err $\pm 0.02 \mathrm{~dB}$ and is insignificant. However, $|R(0)|^{2}=0.6066$ without whitecaps, the difference will cause an erro of about $4.1 \mathrm{~dB}$ in reflected power at normal incident, and will be important for measuring the wind speed on a pulse-limited radar altimeter. So the effect of whitecaps on wind speed retrieval should be considered at a normal incident.

\section{The Improved Wind Speed Model Function at High Sea State Conditions}

\subsection{The Improved Wind Speed Algorithm}

In term of Equation (12) or (21), the wave reflectivity $|R(0)|^{2}$ is a nonlinear function of wind speed. The formation of sea spray droplets and foams is related to the wind speed, which can be represented by the whitecap coverage rate $w_{f}$. The greater the wind speed is, the thicker the droplets layer is, and also the whitecap coverage rate $w_{f}$ becomes biger and biger with increasing the 


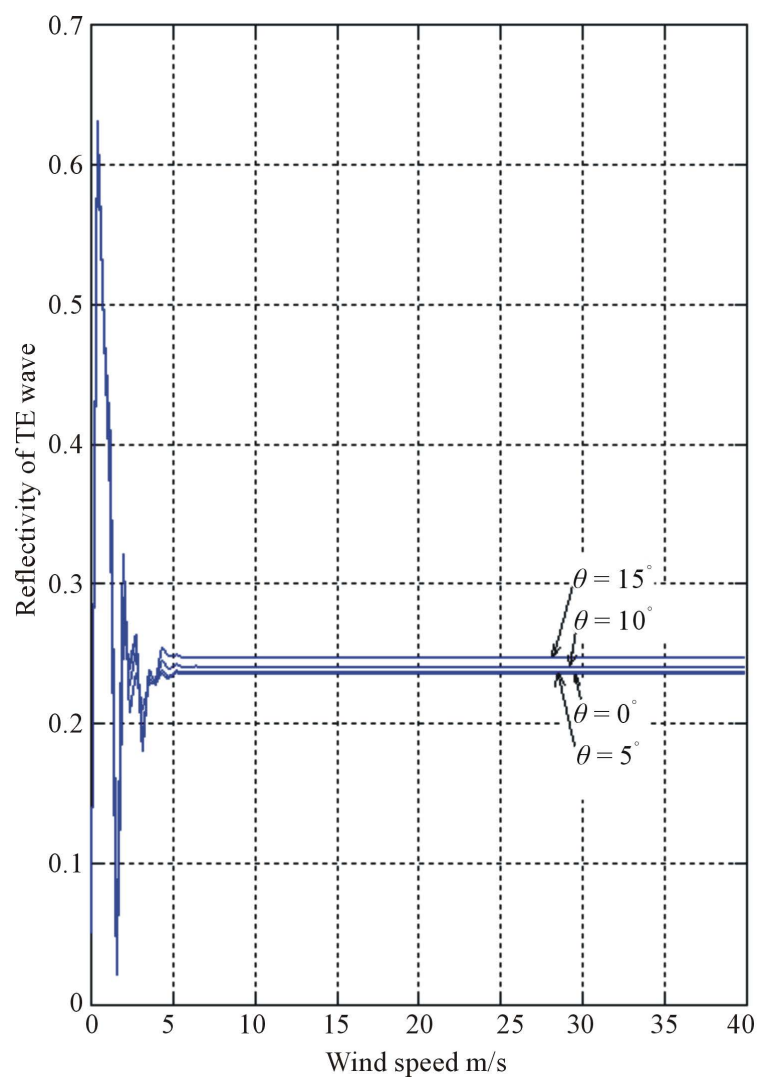

(a)

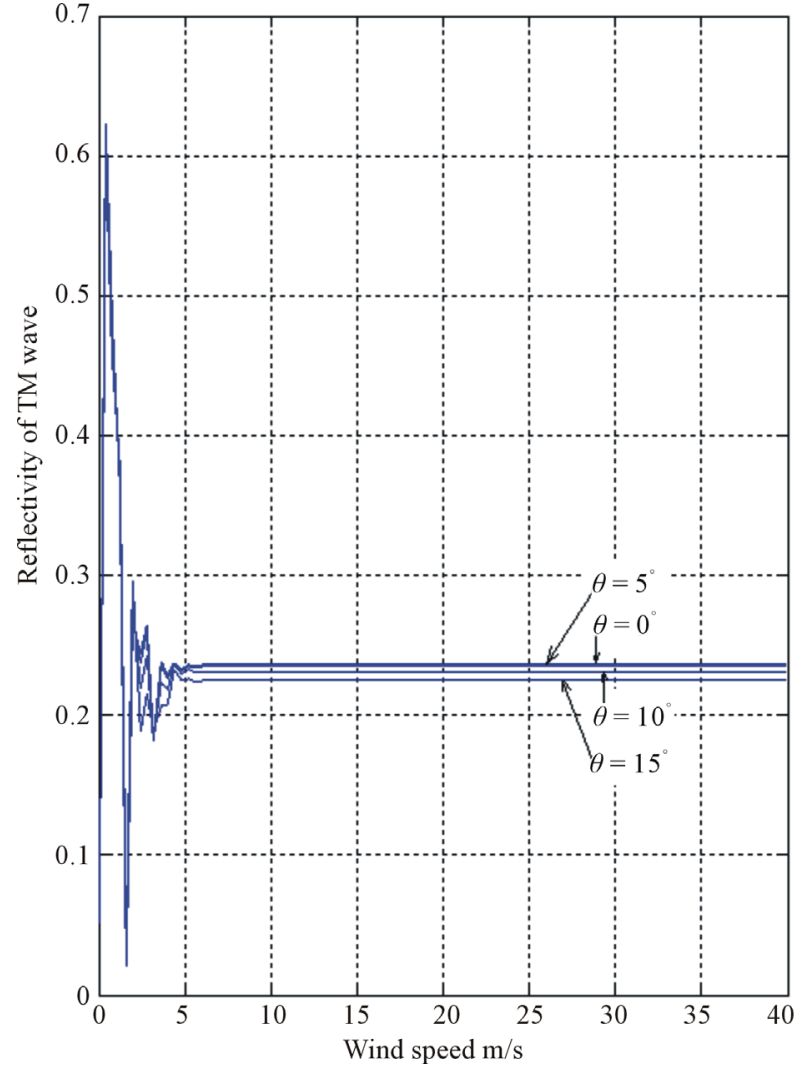

(b)

Figure 2. The reflectivities of TE wave (a) and TM wave (b) versus the wind speed.

wind speed. In 1993, Yeli Yuan et al. carried out a detailed analysis on sea surface broken process, and obtained the analytical expression of whitecap coverage rate $w_{f}$ given as [18].

$$
w_{f}=2.56 \times 10^{-4} H_{s} U_{10}^{1.41}
$$

where $H_{s}$ is the significant wave height, which can be retrieved from the leading curve of the sea surface echo and can also be given as

$$
H_{s}=0.015 U_{10}^{2}
$$

suppose that $|R(0)|^{2}$ is the total sea surface reflectivity factor, $|R|^{2}$ is the reflectivity factor at the interface between the air, droplets and foams layer, $\left|R_{w}\right|^{2}$ is the reflectivity factor at the interface between the air and sea layer. And thus

$$
|R(0)|^{2}=|R|^{2} w_{f}+\left|R_{w}\right|^{2}\left(1-w_{f}\right)
$$

The analytical expressions of ZT wind speed retrieving algorithm $[6,7]$ is

$$
\sigma^{0}=\frac{|R(0)|^{2}}{\alpha} \beta C_{D}^{-1 / 2}\left[2+\frac{3}{2} \ln \frac{a+\sqrt{a^{2}+81 g^{2} /\left(\beta U_{10}\right)^{4}}}{9 g /\left(\beta U_{10}\right)^{2}}-\frac{3}{2} \ln \frac{a+\sqrt{a^{2}+k_{d}^{2}}}{k_{d}}\right]^{-1}
$$

in which $a=0.08, k_{d}=314 \mathrm{~m}^{-1}$ for Ku wave band, $\beta=3.31\left(g H_{s} / U_{10}^{2}\right)^{0.6}, a=\left(g / \gamma_{s}\right)^{1 / 2}$, and $\gamma_{\mathrm{s}}$ is related to sea water density and tension. When the wind speed is more than $2.4 \mathrm{~m} / \mathrm{s}$ we can take [6] $C_{D}=\left(0.8+0.065 U_{10}\right) \times 10^{-3}$. Substituting Equation (24) into Equation (25) we can get

$$
\sigma^{0}=\frac{\left[|R|^{2} w_{f}+\left|R_{w}\right|^{2}\left(1-w_{f}\right)\right] \beta}{\alpha C_{D}^{1 / 2}\left[2+\frac{3}{2} \ln \frac{a+\sqrt{a^{2}+81 g^{2} /\left(\beta U_{10}\right)^{4}}}{9 g /\left(\beta U_{10}\right)^{2}}-\frac{3}{2} \ln \frac{a+\sqrt{a^{2}+k_{d}^{2}}}{k_{d}}\right]}
$$


It is obvious that the effect of spray droplets, foams on electromagnetic wave reflection plays an important role at wind speed retrieving in Equation (26). Ross had some observations of whitecaps in situ and found that when the wind speed was $20 \mathrm{~m} / \mathrm{s}$, the whitecap coverage rate was 21.8\% in the Atlantic Ocean. And when the wind speed was $24.7 \mathrm{~m} / \mathrm{s}$, the whitecap coverage rate in the visible band was 32\% in the North Sea [19]. Obviously, so large a coverage by foam and whitecaps would not be negligible for active microwave remote sensing at high frequencies. Therefore, it is necessary that the reflectivity factor $|R(0)|^{2}$ of the ZT algorithm should be improved to meet with the needs of specific actual sea conditions. Equation (26) is a new improved algorithm presented by the paper.

\subsection{The Analysis of the Improved Wind Speed Algorithm}

For comparison, let us consider the general situation such as wave age $\beta=1$. if the constant $\alpha=0.08$, the derived NRCS from Equation (25) is closest to the satellite NRCS on the condition that $\left|R_{w}(0)\right|^{2}=0.3 .\left|R_{w}(0)\right|^{2}$ is thus taken as $0.3[20,21]$. Figure 3 is a comparison chart of Young algorithm (YG), Brown algorithm (BR), $\mathrm{ZT}$ algorithm and the proposed improvement algorithm. In Figures 3 and 4, we can find some interesting conclusions:

1) At low wind speed $(<20 \mathrm{~m} / \mathrm{s})$, the proposed improvement algorithm can agree with ZT algorithm well. This case is expected because whitecap coverage rate is very small. And the change of $\sigma^{0}$ is so small that it can be negligible.

2) At high wind speeds (20 - $40 \mathrm{~m} / \mathrm{s}$ ), the proposed improvement algorithm and ZT algorithm began to diverge. This case can also be understood because the proposed improvement algorithm fully considered about some effects of the spray droplets, foams on electromagnetic wave reflection while ZT algorithm did not.

3) At high wind speeds (20 - $40 \mathrm{~m} / \mathrm{s}$ ), the proposed improvement algorithm is closer to some experienced algorithms such as Brown-fitted curve and Young-fitted curve in high wind-speed range. And in the $20-30 \mathrm{~m} / \mathrm{s}$ range, the proposed improvement algorithm is agreeable well with Young algorithm (Figure 3). These cases indicate that spray droplets and foams make a contribution to the measurement of radar cross section of the sea surface, and influence the retrieval results of wind speed. The presented algorithms in the paper are valid.

4) Although the effect of foams was considered on measurement results of wind speed [11-13], the plotted curve based on a three-layer model diverged distinctly from YG, BR and ZT especially in high wind speeds (Figure 5). Obviously, the four-layer model in this paper is closer to the practical sea conditions than the threelayer one. It also indicates that the spray droplets layer

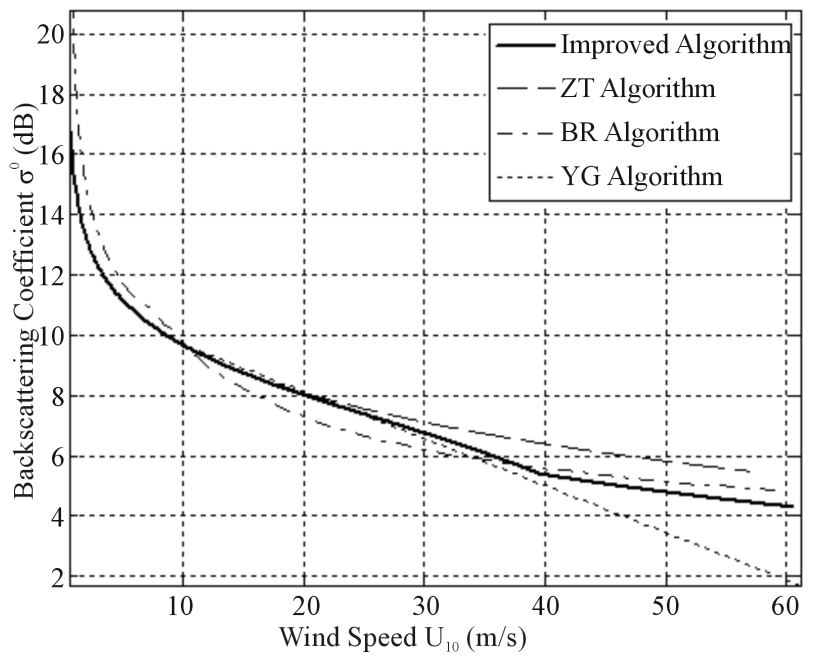

Figure 3. Several algorithms and the new improved algorithm.

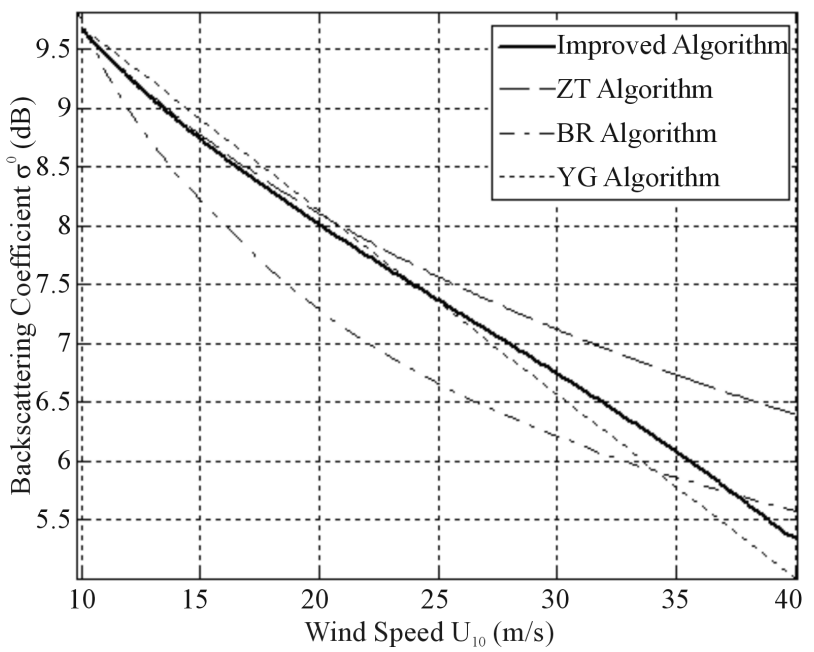

Figure 4. Differences among several functions at 10 - 40 $\mathbf{m} / \mathbf{s}$.

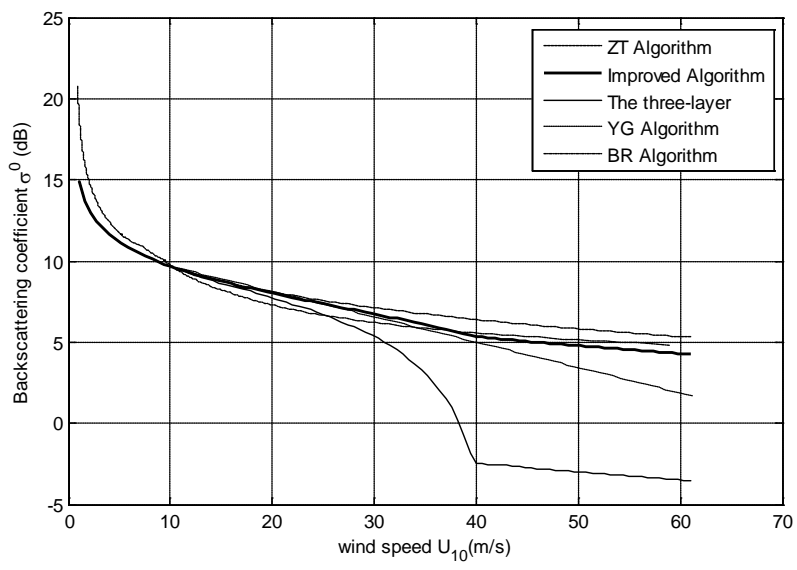

Figure 5. Several algorithms and the new improved algorithm. 
makes a contribution to the measurement result of wind speed. The proposed improvement algorithm based on the four-layer model is closer to actual sea surface practice than ZT.

5) We can draw a conclusion that the proposed improvement algorithm was applicable not only to the low wind speed, but also to the high wind, and also it had a good accuracy.

6) However, there is an inflection point in the vicinity of $40 \mathrm{~m} / \mathrm{s}$ in the proposed improvement algorithm (Figure 3), the phenomenon was because the whitecap coverage rate was close to 1 or more than 1 at $40 \mathrm{~m} / \mathrm{s}$. When the wind speed is more than $40 \mathrm{~m} / \mathrm{s}$, the whitecap coverage rate is 1 , and needed further research.

\section{Summary}

The process of an actual sea state is very complex. In high sea states such as typhoon, the air-sea interaction is very strong. When sea surface wind acts on the waves, the sea waves are broken, and thus bubbles emerge on the sea water surface and the foam layer comes into being, and also on the foam layer there is a spray droplet layer. Foams and droplets have a greater influence on electromagnetic wave reflection. In order to consider about this effect, a four-layer media physical model was established to calculate the microwave reflectivity at 13.5 GHz. Based on the model, an improvement algorithm was presented. The proposed algorithm coincided well with ZT algorithm at low speed wind, but began to diverge at high wind speed. However, the improved algorithm was agreeable with some experienced algorithms such as Young algorithm at the high wind speed (20 - 40 $\mathrm{m} / \mathrm{s}$ ). Compared with ZT algorithm and a three-layer model algorithm, this improved algorithm fully considered the specific sea state, and had good precision in high wind speed retrieving. This algorithm is suitable not only for the low wind speed retrieving, but also for the high wind speed retrieving.

\section{Acknowledgements}

This research was financially supported by the National Natural Science Foundation of China (Grant No. 41076113, Grant NO. 41376181).

\section{REFERENCES}

[1] J. T. McGoogan, L. S. Miller, G. S. Brown and G. S. Hayne, “The S-193 Radar Altimeter Experiment," Proceedings of The IEEE_PIEEE, Vol. 62, No. 6, 1974, pp. 793-803.

[2] H. R. Stanley, “The Geos 3 Project," Journal of Geophysical Research: Solid Earth (1978-2012), Vol. 84, No. B8, 1979, pp. 3779-3783.

[3] D.-L. Zhao and Q. Ye, “On Altimeter wind Speed Model
Functions and Retrieval of Wave Period," Journal of Oceanography, Vol. 26, No. 5, 2004, pp. 1-11.

[4] G. S. Brown, H. R. Stanley and N. A. Roy, "The Wind Speed Measurement Capacity of Space Borne Radar Altimeter," IEEE Journal of Oceanic Engineering, Vol. 6, No. 2, 1981, pp. 59-63.

http://dx.doi.org/10.1109/JOE.1981.1145484

[5] I. R. Young, "An Estimate of the Geosat Altimeter Wind Speed Algorithm at High Wind Speeds," Journal of Geophysical Research, Vol. 98, No. C11, 1993, pp. 2027520285. http://dx.doi.org/10.1029/93JC02117

[6] D. L. Zhao and Y. Toba, "A Spectral Approach for Determining Altimeter Wind Speed Model Functions," Journal of Oceanography, Vol. 59, No. 2, 2003, pp. 235244. http://dx.doi.org/10.1023/A:1025599423405

[7] D. L. Zhao and Y. Toba, "Effect of Wave States on Altimeter Wind Speed Algorithm at High Wind Speeds," The 22nd 267 Asian Conference on Remote Sensing, Singapore City, 5-9 November 2001, pp. 1-6.

[8] V. Karaev and M. Kanevsky, "Determination of the Near Ocean Surface Wind Speed by Altimeter Data: The First Results, Mathematical Methods in Electromagnetic Theory," 6th International Conference on Mathematical Methods in Electromagnetic Theory, Lviv, 10-13 September 1996, pp. 449-452.

[9] J. Gourrion, D. Vandemark, S. Bailey, et al., “A TwoParameter Wind Speed Algorithm for Ku-Band Altimeters,” Journal of Atmospheric and Oceanic Technology, Vol. 19, No. 12, 2002, pp. 2030-2048. http://dx.doi.org/10.1175/1520-0426(2002)019<2030:AT PWSA $>2.0 . \mathrm{CO} ; 2$

[10] F. M. Monaldo and E. B. Dobson, “On Using Significant Wave Height and Radar Cross Section to Improve Radar Altimeter Measurements of Wind Speed," Journal of Geophysical Research, Vol. 94, No. C9, 1989, pp. 1269912701. http://dx.doi.org/10.1029/JC094iC09p12699

[11] Q. Zheng, V. Klemas, G. S. Haynes and N. E. Huang, "The Effect of Oceanic Whitecaps and Foams on PulseLimited Radar Altimeter," Journal of Geophysical Research, Vol. 88, C4, 1983, pp. 2571-2578. http://dx.doi.org/10.1029/JC088iC04p02571

[12] R. M. Gairola and P. C. Pandey, "The Effect of Whitecaps and Foam on Wind Speed Extraction with a Pulse Limited Radar Altimeter," Proceedings of the Indian Academy of Sciences, Earth and Planetary Sciences, Vol.95, No. 2, 1986, pp. 265-273.

[13] L. Yang, J. H. Zou, M. S. Lin and D. L. Pan, "Method to Correct Both Foam and Rain Effects Dual Frequency Altimeter Jason1 Wind Measurements in Typhoon Shanshan,” Proceedings of SPIE, Vol. 7105, 2008, pp. 1-11.

[14] G. R. Valenzuela, "Theories for the Interaction of Electromagnetic and Oceanic Waves-A Review," BoundaryLayer Meteorology, Vol. 13, No. 1-4, 1978, pp. 61-85. http://dx.doi.org/10.1007/BF00913863

[15] D. E. Barrick, "Rough Surface Scattering Based on the Specular Point Theory,” IEEE Transactions on Antennas and Propagation, Vol. AP-16, No. 4, 1968, pp. 449-454. http://dx.doi.org/10.1109/TAP.1968.1139220 
[16] E. L. Andreas and J. B. Edsion, “The Spray Contribution to Net Evaporation from the Sea: A Review of Recent Progress,” Boundary-Layer Meteorology, Vol. 72, No. 1-2, 1995, pp 3-52. http://dx.doi.org/10.1007/BF00712389

[17] J. Wu, “Oceanic Whitecaps and Sea State,” Journal of Physical Oceanography, Vol. 9, No. 5, 1979, pp. 10641068.

[18] E. C. Manahan, et al., "Optimal Power-Law Description of Oceanic Whitecap: Coverage Dependence on Wind Speed," Journal of Physical Oceanography, Vol. 10, No. 12, 1980, pp. 2094-2099. http://dx.doi.org/10.1175/1520-0485(1980)010<2094:OP $\underline{\mathrm{LDOO}>2.0 . \mathrm{CO} ; 2}$

[19] D. B. Ross, et al., "Observations of Oceanic Whitecaps and Their Relation to Remote Sensing Measurements of Surface Wind Speed,” Journal of Geophysical Research, Vol. 79, No. 3, 1974, pp. 444-452. http://dx.doi.org/10.1029/JC079i003p00444

[20] F. C. Jackson, W. T. Walton, D. E. Hines, B. A. Walter, et al., "Sea Surface Mean Square Slope from Ku-Band Backscatter Data,” Journal of Geophysical Research, Vol. 97, No. C7, 1992, pp. 11411-11427.

[21] E. J. Walsh, D. C. Vandemark, C. A. Friehe, S. P. Burns, D. Khelif, R. N. Swift, et al., "Measurements Sea Surface Mean Square Slope with a 36-GHz Scanning Radar Altimeter," Journal of Geophysical Research, Vol. 103, No. C6, 1998, pp. 12587-12601. http://dx.doi.org/10.1029/97JC02443 